Sādhanā, Vol. 19, Part 2, April 1994, pp. 337-346. (C) Printed in India.

\title{
Magneto-visco-elastic surface waves in stressed conducting media
}

\author{
SAMAR CHANDRA DAS ${ }^{1}$, D P ACHARYA $^{2}$ and P R SENGUPTA ${ }^{3}$ \\ ${ }^{1}$ Indian Institute of Mechanics of Continua, 201, Manicktala Main Road, \\ Suite No. 42, Calcutta 700 054, India \\ ${ }^{2}$ Department of Mathematics, Mahadevananda College, Barrackpore, \\ India \\ ${ }^{3}$ Department of Mathematics, University of Kalyani, Kalyani 741235 , \\ India
}

MS received 23 November 1992; revised 9 March 1994

\begin{abstract}
The present paper is concerned with magneto-visco-elastic surface waves in conducting media involving time rate of strain and stress of first order, the media being under an initial stress of hydrostatic tension or compression. The theory of magneto-visco-elastic surface waves in a conducting medium involving time rate of strain and stress of first order is derived under an initial stress. The above general theory is then employed to characterise Rayleigh, Love and Stoneley waves. Results obtained in the above cases reduce to well-known classical results when viscosity and magnetic field are absent.
\end{abstract}

Keywords. Magneto-visco-elastic; first order; surface waves; initial stress; hydrostatic tension or compression.

\section{Introduction}

Surface waves play an important role in the study of earthquakes, seismology, geophysics and geodynamics. The theory of surface waves has been widely investigated and developed by Rayleigh (1885), Voigt (1887), Stoneley (1924), Ewing et al (1957, pp. 257-259, 311), Hunter (1960, pp. 1-57), Bland (1960, pp. 30-75), Flugge (1967, pp. 3-21) and Jeffreys (1959, pp. 35-38). As the inner parts of the earth are under considerable stress from the weight of the matter resting on its surface, we may suppose that the initial equilibrium stress is approximately of hydrostatic nature. Moreover, the earth is placed in its own magnetic-field. Therefore, the investigation presented in this paper may be of importance when surface waves propagate under initial stress, magnetic fields and the viscous nature of the medium are involved. It is believed that the considered problem has not been so far investigated.

The interplay of an electromagnetic field with the motion of deformable solids has also been undertaken by many investigators (Knopoff 1955; Banos 1956; Chadwick 1957; Suhubi 1965; Yu \& Tang 1966; De \& Sengupta 1971). Yu \& Tang (1966) thoroughly discussed the dilatational and rotational waves in a magneto-elastic 
initially stressed conducting medium. De \& Sengupta (1971, 1972) investigated magneto-elastic waves and disturbances in initially stressed conducting media. Acharya \& Sengupta (1978) investigated the problem of magneto-thermo-elastic surface waves in initially stressed conducting media. More recently, the effect of viscosity on the elastic surface waves is receiving greater attention from many investigators (Das \& Sengupta 1990a, 1990b, 1992; Roy \& Sengupta 1983a, 1983b).

In the present paper the authors investigate magneto-visco-elastic surface waves in a conducting medium under hydrostatic stress (tension or compression) paying special attention to Rayleigh, Love and Stoneley waves. Dispersion relations are derived for Rayleigh and Love and some comments on Stoneley waves are also included.

\section{Basic equations}

The equations of motion for a perfectly conducting elastic solid under initial stress (hydrostatic tension or compression) in a uniform magnetic field are (Yu \& Tang 1966)

$$
\begin{gathered}
\rho \frac{\partial^{2} u_{i}}{\partial t^{2}}=-p_{0} \frac{\partial^{2} u_{i}}{\partial x_{j} \partial x_{j}}+\mu_{e} H_{0}\left(\frac{\partial H_{i}}{\partial x_{1}}-\frac{\partial H_{1}}{\partial x_{i}}\right)+\frac{\partial \tau_{i j}}{\partial x_{j}} \\
H_{i}=H_{0}\left(\frac{\partial u_{i}}{\partial x_{1}}-\frac{\partial u_{1}}{\partial x_{i}}\right), \quad i, j=1,2,3
\end{gathered}
$$

where $p_{0}$ is the hydrostatic tension or compression (tension when $p_{0}<0$ and compression when $\left.p_{0}>0\right), \tau_{i j}$ is the stress tensor over the initial stress, $u_{i}$ is the displacement vector with respect to coordinates $x_{1}, x_{2}, x_{3}$ and time $t, \rho$ is the density of the material, $H_{0}$ is the intensity of the uniform magnetic field parallel to $x_{1}$-axis, $\mu_{e}$ is the magnetic permeability.

\section{Formulation of the problem}

Let $M_{1}$ and $M_{2}$ be two electrically conducting charge free isotropic, homogeneous, visco-elastic, semi-infinite solid media in welded contact under an initial hydrostatic

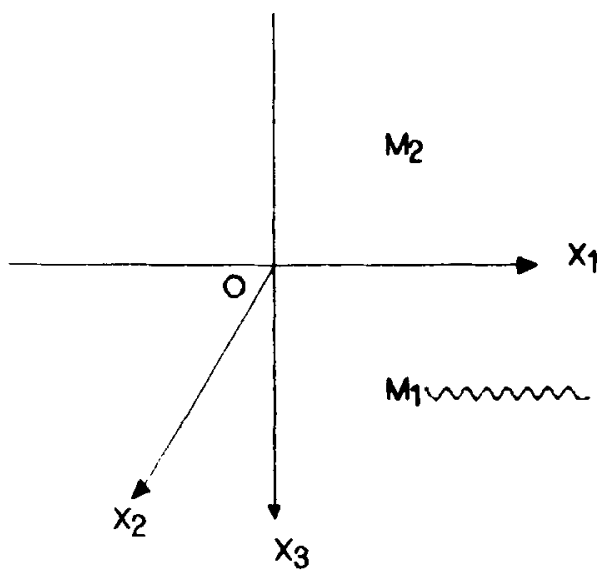

Figure 1. Interface geometry. 
tension or compression permeated by uniform magnetic field (figure 1). We further assume that the medium still remains homogeneous and isotropic under the combined influence of magnetic field and initial stress. The magnetic properties of the conducting solids in the domains $M_{1}$ and $M_{2}$ are assumed to be sensibly the same. We consider a system of orthogonal Cartesian axes $O x_{1} x_{2} x_{3}$, the origin $O$ being on the interface, and $O x_{3}$ being normal to the interface (figure 1 ).

We consider the possibility of a type of wave travelling in the positive $x_{1}$-direction in such a manner that the disturbance is largely confined to the neighbourhood of the boundary and all the particles at any instant on any line parallel to $x_{2}$-axis have equal displacements. Due to the first assumption we assert that the wave is a surface wave and the second assumption concludes that all partial derivatives with respect to the coordinates $x_{2}$ are zero. Then using the formulae $u=\operatorname{grad} \phi+\operatorname{curl} \psi$, the displacement components, $u_{1}$ and $u_{3}$ at any point may be expressed in the form

so that

$$
u_{1}=\frac{\partial \phi}{\partial x_{1}}-\frac{\partial \psi}{\partial x_{3}}, \quad u_{3}=\frac{\partial \phi}{\partial x_{3}}+\frac{\partial \psi}{\partial x_{1}}
$$

$$
\nabla^{2} \phi=\Delta, \quad \nabla^{2} \psi=\frac{\partial u_{3}}{\partial x_{1}}-\frac{\partial u_{1}}{\partial x_{3}}, \quad \nabla^{2}=\frac{\partial^{2}}{\partial x_{1}^{2}}+\frac{\partial^{2}}{\partial x_{3}^{2}}, \quad \Delta=\frac{\partial u_{1}}{\partial x_{1}}+\frac{\partial u_{3}}{\partial x_{3}},
$$

where $\phi$ and $\psi$ are functions of co-ordinates $x_{1}, x_{3}$ and time $t$.

The first order stress-strain relation for an isotropic visco-elastic medium is (Voigt 1887)

$$
\left(\eta_{1}+\eta_{2} \frac{\partial}{\partial t}\right) \tau_{i j}=\left(\lambda_{1}+\lambda_{2} \frac{\partial}{\partial t}\right) \Delta \delta_{i j}+2\left(\mu_{1}+\mu_{2} \frac{\partial}{\partial t}\right) e_{i j}
$$

where $\eta_{1}, \lambda_{1}, \mu_{1}$ are elastic constants, $\eta_{2}, \lambda_{2}$ and $\mu_{2}$ are constants due to viscosity, $e_{i j}=\frac{1}{2}\left(u_{i, j}+u_{j, i}\right)$ is the strain tensor and $\delta_{i j}$ is the Kronecker symbol.

Using (3) in (1), the displacement equations of motion for a conducting first order visco-elastic medium under hydrostatic stress in an uniform magnetic field as

$$
\begin{gathered}
{\left[\left(\lambda_{1}+\mu_{1}\right)+\left(\lambda_{2}+\mu_{2}\right) \frac{\partial}{\partial t}\right] \frac{\partial \Delta}{\partial x_{1}}+\left(\mu_{1}+\mu_{2} \frac{\partial}{\partial t}\right) \nabla^{2} u_{1}} \\
-\left(\eta_{1}+\eta_{2} \frac{\partial}{\partial t}\right) p_{0} \nabla^{2} u_{1}=\rho\left(\eta_{1}+\eta_{2} \frac{\partial}{\partial t}\right) \frac{\partial^{2} u_{1}}{\partial t^{2}} \\
\left(\mu_{1}+\mu_{2} \frac{\partial}{\partial t}\right) \nabla^{2} u_{2}-p_{0}\left(\eta_{1}+\eta_{2} \frac{\partial}{\partial t}\right) \nabla^{2} u_{2}+K\left(\eta_{1}+\eta_{2} \frac{\partial}{\partial t}\right) \frac{\partial^{2} u_{2}}{\partial x_{1}^{2}}= \\
{\left[\left(\lambda_{1}+\mu_{1}\right)+\left(\lambda_{2}+\mu_{2}\right) \frac{\partial}{\partial t}\right] \frac{\partial \Delta}{\partial x_{3}}+\left(\eta_{1}+\eta_{2} \frac{\partial}{\partial t}\right) \frac{\partial^{2} u_{2}}{\partial t^{2}}} \\
+K\left(\eta_{1}+\eta_{2} \frac{\partial}{\partial t}\right)\left(\frac{\partial^{2} u_{3}}{\partial x_{1}^{2}}-\frac{\partial^{2} u_{1}}{\partial x_{1} \partial x_{3}}\right)=\rho\left(\eta_{1}+\eta_{2} \frac{\partial}{\partial t}\right) \frac{\partial^{2} u_{3}}{\partial t^{2}}
\end{gathered}
$$

where $K=\mu_{e} H_{0}^{2}$. The above relations apply to both $M_{1}$ and $M_{2}$. 
Introducing (2) in (4) yields

$$
\begin{aligned}
\frac{\partial^{2} \phi}{\partial t^{2}} & =\left[\frac{\left(V_{1 T}^{2}+V_{2 T}^{2}(\partial / \partial t)\right)}{L}-\frac{p_{0}}{\rho}\right] \nabla^{2} \phi+\frac{K}{\rho} \frac{\partial^{2} \psi}{\partial x_{1} \partial x_{3}}, \\
\frac{\partial^{2} \psi}{\partial t^{2}} & =\left[\frac{\left(V_{1 S}^{2}+V_{2 S}^{2}(\partial / \partial t)\right)}{L}-\frac{p_{0}}{\rho}\right] \nabla^{2} \psi+\frac{K}{\rho} \frac{\partial^{2} \psi}{\partial x_{1}^{2}}, \\
\frac{\partial^{2} u_{2}}{\partial t^{2}} & =\left[\frac{\left(V_{1 S}^{2}+V_{2 S}^{2}(\partial / \partial t)\right)}{L}-\frac{p_{0}}{\rho}\right] \nabla^{2} u_{2}+\frac{K}{\rho} \frac{\partial^{2} u_{2}}{\partial x_{1}^{2}}
\end{aligned}
$$

where

$$
\begin{aligned}
V_{1 T}^{2} & =\frac{\lambda_{1}+2 \mu_{1}}{\rho}, \quad V_{2 T}^{2}=\frac{\lambda_{2}+2 \mu_{2}}{\rho}, \quad V_{1 S}^{2}=\frac{\mu_{1}}{\rho}, \quad V_{2 S}^{2}=\frac{\mu_{2}}{\rho}, \\
L & =\eta_{1}+\eta_{2} \frac{\partial}{\partial t} .
\end{aligned}
$$

Again, (5) apply to both $M_{1}$ and $M_{2}$. In the sequel, $M_{2}$ is identified by $\rho^{\prime}, \eta_{1}^{\prime}, \eta_{2}^{\prime}, \lambda_{1}^{\prime}, \lambda_{2}^{\prime}, \mu_{1}^{\prime}, \mu_{2}^{\prime}$ for its properties.

\subsection{Boundary conditions}

To obtain the frequency equation we apply the following conditions of continuity across the interface

(i) The components of displacement at the interface between $M_{1}$ and $M_{2}$ must be continuous.

(ii) Stress components $\tau_{31}, \tau_{32}, \tau_{33}$ are continuous across the interface. They are respectively given by

$$
\begin{aligned}
& L \tau_{31}=\left(\mu_{1}+\mu_{2} \frac{\partial}{\partial t}\right)\left(2 \frac{\partial^{2} \phi}{\partial x_{1} \partial x_{3}}+\frac{\partial^{2} \psi}{\partial x_{1}^{2}}-\frac{\partial^{2} \psi}{\partial x_{3}^{2}}\right) \\
& L \tau_{32}=\left(\mu_{1}+\mu_{2} \frac{\partial}{\partial t}\right) \frac{\partial u_{2}}{\partial x_{3}} \\
& L \tau_{33}=\left(\lambda_{1}+\lambda_{2} \frac{\partial}{\partial t}\right) \nabla^{2} \phi+2\left(\mu_{1}+\mu_{2} \frac{\partial}{\partial t}\right)\left(\frac{\partial^{2} \phi}{\partial x_{3}^{2}}+\frac{\partial^{2} \psi}{\partial x_{1} \partial x_{3}}\right) .
\end{aligned}
$$

\section{Harmonic solutions}

To investigate equations (5), let us take harmonic solutions

$$
\left(\phi, \psi, u_{2}\right)=\left[\hat{\phi}\left(x_{3}\right), \hat{\psi}\left(x_{3}\right), \hat{u}_{2}\left(x_{3}\right)\right] e^{i\left(\eta x_{1}-\omega t\right)},
$$

for medium $M_{1}$. For medium $M_{2}$, the dashed functions $\hat{\phi}^{\prime}, \hat{\psi}^{\prime}, \hat{u}_{2}^{\prime}$ replace $\hat{\phi}, \hat{\psi}, \hat{u}_{2}$.

Introducing (7) in (5) we get

$$
\frac{d^{2} \hat{\phi}}{d x_{3}^{2}}-\left[\eta^{2}-\frac{\omega^{2} \eta_{K}^{*}}{V_{K T}^{2}-\eta_{K}^{*}\left(p_{0} / \rho\right)}\right] \hat{\phi}=\frac{-i \eta K \eta_{K}^{*}}{\rho\left(V_{K T}^{2}-\left(p_{0} \eta_{K}^{*} / \rho\right)\right)} \frac{d \hat{\psi}}{d x_{3}}
$$




$$
\begin{aligned}
& \frac{d^{2} \hat{\psi}}{d x_{3}^{2}}-\left[\eta^{2}-\frac{\omega^{2} \eta_{K}^{*}}{V_{K S}^{2}-\eta_{K}^{*}\left(p_{0} / \rho\right)}+\frac{K \eta^{2} \eta_{K}^{*}}{\rho\left(V_{K S}^{2}-\eta_{K}^{*}\left(p_{0} / \rho\right)\right.}\right] \hat{\psi}=0, \\
& \frac{d^{2} \hat{u}_{2}}{d x_{3}^{2}}-\left[\eta^{2}+\frac{\left((K / \rho) \eta^{2}-\omega^{2}\right) \eta_{K}^{*}}{V_{K S}^{2}-\left(p_{0} / \rho\right) \eta_{K}^{*}}\right] \hat{u}_{2}=0,
\end{aligned}
$$

in which

$$
\eta_{\mathrm{K}}^{*}=\left(\eta_{1}-i \omega \eta_{2}\right), \quad V_{\mathrm{KT}}^{2}=V_{1 T}^{2}-i \omega V_{2 T}^{2}, \quad V_{K S}^{2}=V_{1 S}^{2}-i \omega V_{2 S}^{2} .
$$

Similar relations for $M_{2}$ can be obtained by using dashed variables $\hat{\phi}^{\prime}, \hat{\psi}^{\prime}, \hat{u}_{2}^{\prime}, \eta_{1}^{\prime}, \eta_{2}^{\prime}$, $V_{1 T}^{\prime}, V_{2 T}^{\prime}, V_{1 S}^{\prime}, V_{2 S}^{\prime}, \eta_{K}^{* \prime}, V_{K T}^{\prime 2}, V_{K S}^{\prime 2}, \lambda_{1}^{\prime}, \mu_{1}^{\prime}, \lambda_{2}^{\prime}, \mu_{2}^{\prime}, \rho^{\prime}$. According to our assumption $K$ is the same for both the media.

Clearly, (8) must have exponential solutions; and, in order that $\phi, \psi$ and $u_{2}$ describe surface waves, they must become vanishingly small as $x_{3}$ tends to infinity. Thus for the medium $M_{1}$ the solutions of (5) may be taken in the following form:

$$
\begin{aligned}
& \phi=\left[A \exp \left\{-x_{3}\left(\eta^{2}-\zeta_{1}^{2}\right)^{1 / 2}\right\}+\beta \exp \left\{-x_{3}\left(\eta^{2}-\zeta_{2}^{2}\right)^{1 / 2}\right\}\right] \exp \left\{i\left(\eta x_{1}-\omega t\right)\right\} \\
& \psi=B_{1} \exp \left\{-x_{3}\left(\eta^{2}-\zeta_{2}^{2}\right)^{1 / 2}+i\left(\eta x_{1}-\omega t\right)\right\} \\
& u_{2}=C \exp \left\{-x_{3}\left[\eta^{2}+\frac{\left.(K / \rho) \eta^{2}-\omega^{2}\right) \eta_{K}^{*}}{V_{K S}^{2}-\left(p_{0} / \rho\right) \eta_{K}^{*}}\right]^{1 / 2}+i\left(\eta x_{1}-\omega t\right)\right\}
\end{aligned}
$$

For the medium $M_{2}$

where

$$
\begin{aligned}
& \phi^{\prime}=\left[A^{\prime} \exp \left\{x_{3}\left(\eta^{2}-\zeta_{1}^{\prime 2}\right)^{1 / 2}\right\}+B^{\prime} \exp \left\{x_{3}\left(\eta^{2}-\zeta_{2}^{\prime 2}\right)^{1 / 2}\right\}\right] \exp \left\{i\left(\eta x_{1}-\omega t\right)\right\} \\
& \psi^{\prime}=B_{1}^{\prime} \exp \left\{x_{3}\left(\eta^{2}-\zeta_{2}^{\prime 2}\right)^{1 / 2}+i\left(\eta x_{1}-\omega t\right)\right\} \\
& u_{2}^{\prime}=C^{\prime} \exp \left\{x_{3}\left[\eta^{2}+\frac{\left(\left(K / \rho^{\prime}\right) \eta^{2}-\omega^{2}\right) \eta_{K}^{* \prime}}{V_{K S}^{\prime 2}-\left(p_{0} / \rho^{\prime}\right) \eta_{K}^{* \prime}}\right]^{1 / 2}+i\left(\eta x_{1}-\omega t\right)\right\}
\end{aligned}
$$

$$
\begin{array}{ll}
\zeta_{1}^{2}=\frac{\omega^{2} \eta_{K}^{*}}{V_{K T}^{2}-\eta_{K}^{*}\left(p_{0} / \rho\right)}, & \zeta_{1}^{2}=\frac{\omega^{2} \eta_{K}^{* \prime}}{V_{K T}^{\prime 2}-\eta_{K}^{* \prime}\left(p_{0} / \rho^{\prime}\right)}, \\
\zeta_{2}^{2}=\frac{\left(\omega^{2}-(K / \rho) \eta^{2}\right) \eta_{K}^{*}}{V_{K S}^{2}-\eta_{K}^{*}\left(p_{0} / \rho\right)}, & \zeta_{2}^{\prime 2}=\frac{\left(\omega^{2}-\left(K \eta^{2} / \rho^{\prime}\right)\right) \eta_{K}^{* \prime}}{V_{K S}^{\prime 2}-\eta_{K}^{* \prime}\left(p_{0} / \rho^{\prime}\right)}
\end{array}
$$

and

with

$$
B=\alpha_{1} B_{1}, \quad B^{\prime}=\alpha_{1}^{\prime} B_{1}^{\prime},
$$

$$
\alpha_{1}=\frac{(i \eta K / \rho)\left(\eta^{2}-\zeta_{2}^{2}\right)^{1 / 2}}{\omega^{2}-\zeta_{2}^{2}\left[\left(V_{K T}^{2} / \eta_{K}^{*}\right)-\left(p_{0} / \rho\right)\right]}, \quad \alpha_{1}^{\prime}=\frac{\left(-i \eta K / \rho^{\prime}\right)\left(\eta^{2}-\zeta_{2}^{\prime 2}\right)^{1 / 2}}{\omega^{2}-\zeta_{2}^{\prime 2}\left[\left(V_{K T}^{\prime 2} / \eta_{K}^{* \prime}\right)-\left(p_{0} / \rho^{\prime}\right)\right]}
$$

In evaluating quantities like $\left(\eta^{2}-\zeta^{2}\right)^{1 / 2}$, the root with positive real part will be taken in each case.

Now applying the boundary conditions (i) and (ii) we get

$$
\begin{aligned}
& A+\left(\alpha_{1}-i Q_{2}\right) B_{1}=A^{\prime}+\left(\alpha_{1}^{\prime}+i Q_{2}^{\prime}\right) B_{1}^{\prime}, \\
& C=C^{\prime}, \\
& i Q_{1} A+\left(i Q_{2} \alpha_{1}+1\right) B_{1}=-i Q_{1}^{\prime} A^{\prime}-\left(i \alpha_{1}^{\prime} Q_{2}^{\prime}-1\right) B_{1}^{\prime},
\end{aligned}
$$




$$
\begin{aligned}
& \begin{aligned}
& \rho\left(\frac{V_{K S}^{2}}{\eta_{K}^{*}}\right) {\left[2 i Q_{1} A+\left\{\left(1+Q_{2}^{2}\right)+2 i Q_{2} \alpha_{1}\right\} B_{1}\right] } \\
&=\rho^{\prime}\left(\frac{V_{K S}^{\prime 2}}{\eta_{K}^{* \prime}}\right)\left[-2 i Q_{1}^{\prime} A^{\prime}+\left\{-2 i Q_{2}^{\prime} \alpha_{1}^{\prime}+\left(1+Q_{2}^{\prime 2}\right)\right\} B_{1}^{\prime}\right] \\
&-\rho\left(\frac{V_{K S}^{2}}{\eta_{K}^{*}}\right)\left\{\eta^{2}+\frac{\left((K / \rho) \eta^{2}-\omega^{2}\right) \eta_{K}^{*}}{V_{K S}^{2}-\left(p_{0} / \rho\right) \eta_{K}^{*}}\right\}^{1 / 2} C \\
&=\rho^{\prime}\left(\frac{V_{K S}^{\prime 2}}{\eta_{K}^{* \prime}}\right)\left\{\eta^{2}+\frac{\left(\left(K / \rho^{\prime}\right) \eta^{2}-\omega^{2}\right) \eta_{K}^{* \prime}}{\left(V_{K S}^{\prime 2}-\left(p_{0} / \rho^{\prime}\right) \eta_{K}^{* \prime}\right)}\right\}^{1 / 2} C^{\prime} \\
&\left(\frac{\rho}{\eta_{K}^{*}}\right)\left[\left\{V_{K T}^{2}\left(Q_{1}^{2}-1\right)+2 V_{K S}^{2}\right\} A+\left\{V_{K T}^{2}\left(Q_{2}^{2}-1\right) \alpha_{1}+2 V_{K S}^{2}\left(\alpha_{1}-i Q_{2}\right)\right\} B_{1}\right] \\
&=\left(\frac{\rho^{\prime}}{\eta_{K}^{* \prime}}\right)\left[\left\{V_{K T}^{\prime 2}\left(Q_{1}^{\prime 2}-1\right)+2 V_{K S}^{\prime 2}\right\} A^{\prime}+\right. \\
&\left.\quad+\left\{V_{K T}^{\prime 2}\left(Q_{2}^{\prime 2}-1\right) \alpha_{1}^{\prime}+2 V_{K S}^{\prime 2}\left(\alpha_{1}^{\prime}+i Q_{2}^{\prime}\right)\right\} B_{1}^{\prime}\right]
\end{aligned}
\end{aligned}
$$

where

$$
Q_{k}=\left(1-\frac{\zeta_{K}^{2}}{\eta^{2}}\right)^{1 / 2}, \quad Q_{K}^{\prime}=\left(1-\frac{\zeta_{K}^{2}}{\eta^{2}}\right)^{1 / 2}, . \quad k=1,2
$$

From (12b) and (12e) we get

$$
C=C^{\prime}=0 \text {. }
$$

Thus we conclude that there is no propagation of the displacement $u_{2}$. The wave velocity equation is, therefore, obtained from $(12 \mathrm{a}),(12 \mathrm{c}),(12 \mathrm{~d}),(12 \mathrm{f})$ by the elimination of the constants $A, B_{1}, A^{\prime}, B_{1}^{\prime}$, in the determinant form as

$$
\left|M_{i j}\right|=0, \quad(i, j=1,2,3,4) \text {, }
$$

where

$$
\begin{aligned}
& M_{11}=1, \quad M_{12}=\left(\alpha_{1}-i Q_{2}\right), \quad M_{13}=-1, \quad M_{14}=-\left(\alpha_{1}^{\prime}+i Q_{2}^{\prime}\right), \\
& M_{21}=i Q_{1}, \quad M_{22}=\left(i Q_{2} \alpha_{1}+1\right), \quad M_{23}=i Q_{1}^{\prime}, \quad M_{24}=\left(i \alpha_{1}^{\prime} Q_{2}^{\prime}-1\right), \\
& M_{31}=\rho\left(V_{K S}^{2} / \eta_{K}^{*}\right) 2 i Q_{1}, \quad M_{32}=\rho\left(V_{K S}^{2} / \eta_{K}^{*}\right)\left\{\left(1+Q_{2}^{2}\right)+2 i Q_{2} \alpha_{1}\right\}, \\
& M_{33}=\rho^{\prime}\left(V_{K S}^{\prime 2} / \eta_{K}^{* \prime}\right) 2 i Q_{1}^{\prime}, \quad M_{34}=-\rho^{\prime}\left(V_{K S}^{\prime 2} / \eta_{K}^{* \prime}\right)\left\{-2 i Q_{2}^{\prime} \alpha_{1}^{\prime}+\left(1+Q_{2}^{\prime 2}\right)\right\}, \\
& M_{41}=\left(\rho / \eta_{K}^{*}\right)\left\{V_{K T}^{2}\left(Q_{1}^{2}-1\right)+2 V_{K S}^{2}\right\} \\
& M_{42}=\left(\rho / \eta_{K}^{*}\right)\left\{V_{K T}^{2}\left(Q_{2}^{2}-1\right) \alpha_{1}+2 V_{K S}^{2}\left(\alpha_{1}-i Q_{2}\right)\right\}, \\
& M_{43}=-\left(\rho^{\prime} / \eta_{K}^{* \prime}\right)\left\{V_{K T}^{\prime 2}\left(Q_{1}^{\prime 2}-1\right)+2 V_{K S}^{\prime 2}\right\}, \\
& M_{44}=-\left(\rho^{\prime} / \eta_{K}^{* \prime}\right)\left\{V_{K T}^{\prime 2}\left(Q_{2}^{\prime 2}-1\right) \alpha_{1}^{\prime}+2 V_{K S}^{\prime 2}\left(\alpha_{1}^{\prime}+i Q_{2}^{\prime}\right)\right\}
\end{aligned}
$$

From (13) we obtain the wave velocity in the common boundary under consideration in the presence of magnetic field, initial stress in the nature of hydrostatic tension or compression, viscosity where viscosity is of first order including strain rate and stress rate. 


\section{Particular cases}

\subsection{Rayleigh waves}

In the particular case of Rayleigh waves the interface becomes a free surface and $M_{2}$ is treated as vacuum.

Hence in view of $(12 d)$ and $(12 f)$ we get

$$
\begin{aligned}
& 2 i Q_{1} A+\left\{\left(1+Q_{2}^{2}\right)+2 i Q_{2} \alpha_{1}\right\} B_{1}=0, \\
& \left\{V_{K T}^{2}\left(Q_{1}^{2}-1\right)+2 V_{K S}^{2}\right\} A+\left\{V_{K T}^{2}\left(Q_{2}^{2}-1\right) \alpha_{1}+2 V_{K S}^{2}\left(\alpha_{1}-i Q_{2}\right)\right\} B_{1}=0 .
\end{aligned}
$$

For the indispensable constants $A, B_{1}$ from (14) and (15) to assume non zero values we have

where

$$
\left|M_{i j}^{\prime}\right|=0 \quad(i, j=1,2)
$$

$$
\begin{aligned}
& M_{11}^{\prime}=2 i Q_{1}, \quad M_{12}^{\prime}=\left(1+Q_{2}^{2}\right)+2 i Q_{2} \alpha_{1}, \\
& M_{21}^{\prime}=V_{K T}^{2}\left(Q_{1}^{2}-1\right)+2 V_{K S}^{2}, \quad M_{22}^{\prime}=V_{K T}^{2}\left(Q_{2}^{2}-1\right) \alpha_{1}+2 V_{K S}^{2}\left(\alpha_{1}-i Q_{2}\right) .
\end{aligned}
$$

Equation (16) represents the magneto-visco-elastic Rayleigh wave velocity equation under the initial stress in the nature of hydrostatic tension or compression in a medium, including strain rate and stress rate.

In the absence of viscous effects, (16) reduces to

$$
\left|\begin{array}{ll}
2 i P_{1} & \left(1+P_{2}^{2}\right)+2 i n_{1} P_{2} \\
V_{1 T}^{2}\left(P_{1}^{2}-1\right)+2 V_{1 S}^{2} & V_{1 T}^{2}\left(P_{2}^{2}-1\right) n_{1}+2 V_{1 S}^{2}\left(n_{1}-i P_{2}\right)
\end{array}\right|=0
$$

where

$$
\begin{aligned}
& P_{1}^{2}=1-\frac{\omega^{2}}{\left(V_{1 T}^{2}-\left(p_{0} / \rho\right)\right) \eta^{2}}, \quad P_{2}^{2}=1-\frac{\omega^{2}-K \eta^{2} / \rho}{\left(V_{1 S}^{2}-\left(p_{0} / \rho\right)\right) \eta^{2}} \\
& n_{1}=\frac{i K\left[1-\left(\omega^{2} / \eta^{2} V_{1 S}^{2}\right)+\left(K / \rho V_{1 S}^{2}\right)\right]^{1 / 2}}{\rho V_{1 T}^{2}\left[\left(\omega^{2} / \eta^{2} V_{1 T}^{2}\right)-\left(\omega^{2} / \eta^{2} V_{1 S}^{2}\right)+\left(K V_{1 T}^{2} / \rho V_{1 S}^{2}\right)\right]}
\end{aligned}
$$

Equation (17) represents the magneto-elastic Rayleigh-wave velocity equation under the initial stress of hydrostatic tension or compression. This agrees with the result obtained by Acharya \& Sengupta (1978).

Moreover, in the absence of the magnetic field and initial stress $\left(p_{0}=0, K=0\right)$ we get from (17) the Rayleigh wave velocity equation for the elastic medium as

where

$$
4\left[1-\left(c^{2} / V_{1 T}^{2}\right)\right]^{1 / 2}\left[1-\left(c^{2} / V_{1 S}^{2}\right)\right]^{1 / 2}=\left(2-\left(c^{2} / V_{1 S}^{2}\right)\right)^{2},
$$

$$
c^{2}=\omega^{2} / \eta^{2}
$$

Equation (18) is in complete agreement with the classical result of Rayleigh.

\subsection{Love waves}

We know that for such types of waves $u_{2}$ is the only component of displacement vector $u$ to play the role. Let us consider that the medium $M_{2}$ is bounded by two 


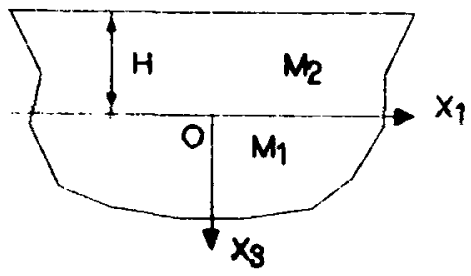

Figure 2. Love wave formulation.

horizontal plane surfaces at a finite distance $H$ apart, the upper plane surface being free while the medium $M_{1}$ extends to an infinitely great distance (figure 2).

The notable fact here is that the displacement in $M_{2}$ may no longer diminish with distance from the boundary between $M_{1}$ and $M_{2}$ so that for the medium $M_{2}$ we preserve the full solution as

$$
\begin{aligned}
u_{2}^{\prime}= & {\left[C_{1}^{\prime} \exp \left\{x_{3}\left(\eta^{2}+\frac{\left(\left(K / \rho^{\prime}\right) \eta^{2}-\omega^{2}\right) \eta_{K}^{* \prime}}{V_{K S}^{\prime 2}-\left(p_{0} / \rho^{\prime}\right) \eta_{K}^{* \prime}}\right)^{1 / 2}\right\}+\right.} \\
& \left.C_{2}^{\prime} \exp \left\{-x_{3}\left(\eta^{2}+\frac{\left(\left(K \eta^{2} / \rho^{\prime}\right)-\omega^{2}\right) \eta_{K}^{* \prime}}{\left(V_{K S}^{\prime 2}-\left(p_{0} / \rho^{\prime}\right) \eta_{K}^{* \prime}\right)}\right)^{1 / 2}\right\}\right] \times \exp \left(i\left(\eta x_{1}-\omega t\right)\right),
\end{aligned}
$$

where the restriction that the real part of $\left\{\eta^{2}+\left(\left(K / \rho^{\prime}\right) \eta^{2}-\omega^{2}\right) \eta_{K}^{* \prime} /\left[V_{K S}^{\prime 2}-\left(p_{0} / \rho^{\prime}\right) \eta_{K}^{* \prime}\right]\right\}^{1 / 2}$ be positive is not required.

For the present case the boundary conditions are

(i) $u_{2}$ and $\tau_{32}$ are continuous at $x_{3}=0$,

(ii) $\tau_{32}^{\prime}=0$ at $x_{3}=-H$.

Employing the boundary conditions (i) and (ii) we obtain

$$
\begin{aligned}
& C=C_{1}^{\prime}+C_{2}^{\prime} \\
& -\rho\left(\frac{V_{K S}^{2}}{\eta_{K}^{*}}\right)\left[\eta^{2}+\frac{\left(\left(K \eta^{2} / \rho\right)-\omega^{2}\right) \eta_{K}^{*}}{V_{K S}^{2}-\left(p_{0} / \rho\right) \eta_{K}^{*}}\right]^{1 / 2} C=\rho^{\prime}\left(\frac{V_{K S}^{\prime 2}}{\eta_{K}^{* \prime}}\right) \\
& \times\left[\eta^{2}+\frac{\left(\left(K \eta^{2} / \rho^{\prime}\right)-\omega^{2}\right) \eta_{K}^{* \prime}}{V_{K S}^{\prime 2}-\left(p_{0} / \rho^{\prime}\right) \eta_{K}^{* \prime}}\right]^{1 / 2}\left(C_{1}^{\prime}-C_{2}^{\prime}\right), \\
& C_{1}^{\prime} e^{-H}\left[\eta^{2}+\frac{\left(\left(K / \rho^{\prime}\right) \eta^{2}-\omega^{2}\right) \eta_{K}^{* \prime}}{V_{K S}^{\prime 2}-\left(p_{0} / \rho^{\prime}\right) \eta_{K}^{* \prime}}\right]^{1 / 2} \\
& -C_{2}^{\prime} e^{-H}\left[\eta^{2}+\frac{\left(\left(K \eta^{2} / \rho^{\prime}\right)-\omega^{2}\right) \eta_{K}^{* \prime}}{V_{K S}^{\prime 2}-\left(p_{0} / \rho^{\prime}\right) \eta_{K}^{* \prime}}\right]^{1 / 2}=0 .
\end{aligned}
$$

Eliminating $C, C_{1}^{\prime}, C_{2}^{\prime}$ from equations (20a)-(20c) we get

$$
\begin{gathered}
\rho\left(\frac{V_{K S}^{2}}{\eta_{K}^{*}}\right)\left[1-\frac{\left(c^{2}-(K / \rho)\right) \eta_{K}^{*}}{V_{K S}^{2}-\left(p_{0} / \rho\right) \eta_{K}^{*}}\right]^{1 / 2}-\rho^{\prime}\left(\frac{V_{K S}^{\prime 2}}{\eta_{K}^{* \prime}}\right)\left[\frac{\left(c^{2}-\left(K / \rho^{\prime}\right)\right) \eta_{K}^{* \prime}}{V_{K S}^{\prime 2}-\left(p_{0} / \rho^{\prime}\right) \eta_{K}^{* \prime}}-1\right]^{1 / 2} \\
\times \tan \left\{\eta H\left[\frac{\left(c^{2}-\left(K / \rho^{\prime}\right)\right) \eta_{K}^{* \prime}}{V_{K S}^{\prime 2}-\left(p_{0} / \rho^{\prime}\right) \eta_{K}^{* \prime}}-1\right]^{1 / 2}\right\}=0
\end{gathered}
$$

where $c=\omega / \eta$. 
Equation (21) represents the required wave velocity equations for Love waves in a magneto-visco-elastic solid medium including strain and stress rates of first order under an initial hydrostatic tension or compression. It is seen from (21) that Love waves depend upon viscous field as well as magnetic field and also on the initial stress in the nature of hydrostatic tension or compression.

\subsection{Stoneley waves}

In the classical theory, Stoneley waves are the generalised form of Rayleigh waves propagating in the vicinity of interface of two semi-infinite media $M_{1}$ and $M_{2}$. Hence in our general case Stoneley waves propagating along the common boundary of $M_{1}$ and $M_{2}$ are determined by the roots of the wave velocity equation (13). In the absence of magnetic field, initial stress, viscosity and strain-stress rates this equation of course reduces to the classical result obtained by Stoneley.

We are grateful to the referees for their valuable comments and suggestions towards improvement of the paper and its appearance in the present form.

\section{References}

Acharya D P, Sengupta P R 1978 Magneto-thermo-elastic surface waves in initially stressed conducting media. Acta Geophys. Polon. 26(4):

Banos A 1956 Normal modes characterising magneto-elastic plane waves. J. Phys. Rev. 104: 300-305

Bland D R 1960 The theory of linear visco-elasticity (London: Pergamon) (This monograph on the subject contains many cases of stress analysis)

Chadwick P 1957 Elastic wave propagation in a magnetic field. IX Congress Int. Mech. Appl. 7: 143-158

Das T K, Sengupta P R 1990a Surface waves in general viscoelastic media of higher order. Indian. J. Pure Appl. Math. 21(7): 661-675

Das T K, Sengupta P R 1990b Surface waves in thermo-visco-elastic media considering time rate of stress and strain of higher order. Gerlands Beitr. Geophys. Leipzig 99: 337-448

Das T K, Sengupta P R 1992 Effect of gravity on visco-elastic surface waves in solids involving time rate of strain and stress of first order. Sädhanā 17: 315-323

De S N, Sengupta P R 1971 Surface waves in magneto-elastic initially stressed conducting media. Pure Appl. Geophys. 88: 44-52

De S N, Sengupta P R 1972 Magneto-elastic waves and disturbances in initially stressed conducting media. Pure Appl. Geophys. 93: 41-54

Ewing W M, Jardetzky W S, Press F 1957 Elastic waves in layered media (London: McGraw-Hill)

Flugge W 1967 Visco-elasticity (London: Blaisdell)

Hunter S C 1960 Visco-elastic waves. Progress in solid mechanics (eds) I N Snedon, R Hill (Amsterdam, New York: North Interscience)

Jeffreys H 1959 The earth 4th edn (Cambridge: University Press)

Knopoff L 1955 The interaction between elastic wave motions and a magnetic field in electric conductors. J. Geophys. Res. 60: 441-456

Rayleigh Lord 1885 On waves propagated along the plane surface of an elastic solid. Proc. London Math. Soc. 17: 4-11

Roy S K, Sengupta P R 1983a Rotatory vibration of a sphere of general visco-elastic solid. Gerlands Beitr. Geophys. Leipzig 92: 70-76

Roy S K, Sengupta P R 1983b Radial vibration of a sphere of general visco-elastic solid. Gerlands Beitr. Geophys. Leipzig 92: 435-442 
Stoneley R 1924 Elastic waves at the surface of separation of two solids. Proc. R. Soc., London 106: 416-428

Suhubi E S 1965 Small torsional oscillations of a circular cylinder with finite electric conductivity in a constant axial magnetic field. Int. J. Eng. Sci. 2: 441-459

Voigt W 1887 Theoretische Studien uber die Elasticitats Verhaltnisse der Krystalle. Abh. Ges. Wiss. Gottingen 34

Yu C P, Tang S 1966 Magneto-elastic waves in initially stressed conductors. Z. Angew. Math. Phys. 17: 766 